\title{
Efecto de la localización del electrodo ventricular izquierdo sobre los parámetros ecocardiográficos de asincronía en pacientes sometidos a terapia de resincronización cardíaca
}

\author{
Alfonso Macías, Juan J. Gavira, Eduardo Alegría, Pedro M. Azcárate, Joaquín Barba \\ e Ignacio García-Bolao
}

Departamento de Cardiología y Cirugía Cardiovascular. Clínica Universitaria de Navarra.

Facultad de Medicina. Universidad de Navarra. Pamplona. España.

Introducción y objetivos. La terapia de resincronización ha mostrado su eficacia como alternativa al tratamiento de pacientes con insuficiencia cardíaca. Las indicaciones actuales se basan en criterios clínicos y electrocardiográficos, aunque la ecocardiografía es una herramienta útil para el diagnóstico de asincronía. El objetivo de este estudio es valorar la asincronía ventricular mediante ecocardiografía, así como el efecto resincronizador según la localización del electrodo ventricular izquierdo.

Pacientes y método. Se estudió a los primeros 25 pacientes (13 con el electrodo en posición lateral y 12, anterior) sometidos a terapia de resincronización. Se valoraron ecocardiográficamente, además de la fracción de eyección y los diámetros ventriculares, los parámetros de asincronía inter e intraventricular antes del implante y a los 3 meses, con el dispositivo conectado y desconectado.

Resultados. La fracción de eyección mejoró de 23,7 \pm $6,5 \%$ hasta $27,8 \pm 5,5 \%$ a los 3 meses ( $p=0,007)$. La estimulación biventricular en cualquier localización se asoció a una mejora significativa de todos los parámetros de asincronía intraventricular (retraso septo-pared posterior y retraso septo-pared lateral). Se observó una disminución significativa, tanto del retraso septo-pared posterior $(58,1$ frente a $118 \mathrm{~ms} ; \mathrm{p}=0,02)$ como del retraso septopared lateral $(39,5$ frente a $86,5 \mathrm{~ms} ; \mathrm{p}=0,04)$, en los pacientes con el electrodo en posición lateral en relación con aquellos con el electrodo en posición anterior. Tres pacientes, todos con el electrodo en posición anterior, fueron considerados no respondedores.

Conclusiones. La estimulación desde la región lateral del ventrículo izquierdo se asocia a una mejor corrección de los parámetros de asincronía intraventricular que la estimulación desde la zona anterior. La ecocardiografía es una técnica útil para valorar los cambios de la sincronía intra e interventricular según las diferentes zonas estimuladas.

Palabras clave: Insuficiencia cardíaca. Terapia de resincronización cardíaca. Estimulación biventricular. Marcapasos. Ecocardiografía.

Correspondencia: Dr. I. García-Bolao.

Departamento de Cardiología y Cirugía Cardiovascular.

Clínica Universitaria de Navarra.

Avda. Pio XII, s/n. 31008 Pamplona. España.

Correo electrónico: igarciab@unav.es

Recibido el 10 de julio de 2003

Aceptado para su publicación el 28 de noviembre de 2003.

\section{Effect of the Left Ventricular Pacing Site on Echocardiographic Parameters of Ventricular Dyssynchrony in Patients Receiving Cardiac Resynchronization Therapy}

Introduction and objectives. Cardiac resynchronization therapy has been shown to be an option in the treatment of patients with congestive heart failure. The current indication for this treatment is based on clinical and electrocardiographic criteria, although echocardiography has also been shown to be a useful tool for the diagnosis of ventricular dyssynchrony. The aim of this study was to assess left ventricular dyssynchrony by echocardiography and to evaluate the effect of the stimulation site on the magnitude of resynchronization.

Patients and method. We studied 25 patients with biventricular stimulation (left ventricular lead located in a lateral position in 13 patients, and in an anterior position in 12). A complete echo-Doppler evaluation, including left ventricular ejection fraction, ventricular diameters and parameters of inter- and intraventricular dyssynchrony, was performed before implantation and 3 months after the procedure, with the device connected and disconnected.

Results. Left ventricular ejection fraction increased significantly from $23.7(6.5)$ to $27.8(5.5) \quad(P=.007)$ at 3 months. In the group as a whole, biventricular pacing was associated with a significant decrease in all intraventricular dyssynchrony parameters (septal-to-lateral wall motion delay and septal-to-posterior wall motion delay). This decrease in septal-to-posterior wall motion delay and septalto-lateral wall motion delay was significantly greater in patients with the electrode implanted in the lateral position ( $58.1 \mathrm{~ms}$ vs $118 \mathrm{~ms} ; P=.02)$ than with the lead in the anterior position (39.5 ms vs $86.5 \mathrm{~ms} ; P=.04$ ). Three patients, all with the electrode in an anterior location, were considered non-responders.

Conclusions. Left lateral free wall stimulation provided significantly better intraventricular resynchronization compared to stimulation at an anterior site. Echocardiography is a useful tool to evaluate changes in intra- and interventricular synchrony related to the pacing site.

Key words: Heart failure. Cardiac resynchronization therapy. Biventricular pacing. Pacemaker. Echocardiography.

Full English text available at: www.revespcardiol.org 


\section{ABREVIATURAS}

TRC: terapia de resincronización cardíaca.

ECG: electrocardiograma.

RSP: retraso septo-pared posterior.

RSL: retraso septo-pared lateral.

\section{INTRODUCCIÓN}

La terapia de resincronización cardíaca (TRC) mediante estimulación auriculobiventricular se ha convertido en una prometedora herramienta para el tratamiento de pacientes con insuficiencia cardíaca avanzada, disfunción ventricular sistólica severa y trastornos de la conducción intra e interventricular, fundamentalmente debidos a bloqueo de la rama izquierda del haz de His ${ }^{1}$.

Diversos ensayos clínicos han puesto de manifiesto un beneficio de esta técnica en términos de mejoría de la clase funcional y del consumo máximo de oxígeno, disminución del número de ingresos hospitalarios por descompensación de la enfermedad y aumento de la fracción de eyección ventricular izquierda ${ }^{2-5}$. Sin embargo, en dichos estudios se ha observado que hasta un $30 \%$ de los pacientes no responde a la TRC. Este hecho puede ser debido tanto a una imperfecta selección de los pacientes como a una inadecuada posición final del electrodo ventricular izquierdo ${ }^{6}$.

En cuanto a la selección de potenciales candidatos a recibir esta terapia, actualmente se realiza sobre la base de criterios clínicos (grado funcional III o IV de la New York Heart Association [NYHA]) y electrocardiográficos (trastorno de la conducción intraventricular, sobre todo por bloqueo de rama izquierda, con un complejo QRS superior a $120 \mathrm{~ms})^{7}$. Estos criterios se basan en la teoría de que el retraso electromecánico intra e interventricular, y por tanto la asincronía ventricular, estaría producido por el bloqueo de rama izquierda ${ }^{8}$. Sin embargo, no está claro que la duración del complejo QRS muestre una adecuada correlación con la magnitud de la asincronía, y mucho menos que sea un predictor de respuesta favorable. Por este motivo, se está intentando aplicar otras alternativas diagnósticas más específicas en la valoración de la asincronía inter e intraventricular. Aunque hasta el momento no existe una validación universal de los criterios ecocardiográficos en el diagnóstico de la asincronía o en la posible predicción de una respuesta favorable a la TRC, la ecocardiografía en sus diversas modalidades técnicas es, en ambos sentidos, una exploración prometedora que presenta indudables ventajas, como su carácter no invasivo y la posibilidad de realizar varios estudios en el seguimiento de un mismo paciente ${ }^{9}$.
Respecto a la posición ideal del electrodo ventricular izquierdo para conseguir un máximo efecto resincronizador, alcanzar una localización óptima durante el implante no resulta fácil en muchas ocasiones. Esto puede ser debido a peculiaridades de la anatomía venosa coronaria, a la imposibilidad de obtener parámetros eléctricos aceptables en la zona deseada o a dificultades de tipo técnico ${ }^{10}$. En cualquier caso, se ha identificado la posición lateral o posterolateral del ventrículo izquierdo como la que se asocia a un mayor beneficio hemodinámico agudo, sobre todo en lo que se refiere al aumento del $\mathrm{dp} / \mathrm{dt}^{11-13}$. Sin embargo, hasta el momento no se ha comprobado el efecto de la posición del electrodo ventricular izquierdo sobre los parámetros ecocardiográficos de resincronización.

El objetivo del presente estudio es valorar la asincronía intra e interventricular mediante ecocardiografía-Doppler antes y a los 3 meses de seguimiento tras el implante de un dispositivo de estimulación auriculobiventricular, así como cuantificar la magnitud del efecto resincronizador en relación con la posición final del electrodo ventricular izquierdo en pacientes con insuficiencia cardíaca avanzada sometidos a TRC.

\section{PACIENTES Y MÉTODO}

\section{Población del estudio}

De una población total de 49 pacientes sometidos a TRC se incluyó a los 13 primeros casos en los que se había implantado el electrodo ventricular izquierdo en posición lateral y a los 12 primeros con el electrodo implantado en posición anterior. Todos ellos presentaban insuficiencia cardíaca crónica en grado funcional III o IV de la NYHA a pesar del tratamiento médico máximo tolerado, disfunción ventricular izquierda (fracción de eyección $<35 \%$ ), ritmo sinusal y bloqueo de rama izquierda con una duración del complejo QRS $>120$ ms. Se excluyó del estudio a los pacientes con insuficiencia cardíaca aguda, con valvulopatía o cardiopatía isquémica susceptibles de tratamiento quirúrgico, con indicación para marcapasos convencional y a los que presentaban fibrilación auricular permanente.

\section{Protocolo del estudio}

Antes de la implantación del dispositivo se realizaron las siguientes pruebas a todos los pacientes: historia clínica, exploración física, electrocardiograma (ECG) de 12 derivaciones y un ecocardiogramaDoppler. Las mismas exploraciones se repitieron a los 3 meses de seguimiento en todos los pacientes, y en el caso del ecocardiograma, se realizaron las mediciones con y sin resincronización. La máxima duración del complejo QRS se midió en las derivaciones II, V1 y V6 del ECG. 


\section{Estudio ecocardiográfico}

El estudio ecocardiográfico (Sonos 5500, HewlettPackard, sonda de $3 \mathrm{MHz}$ ) se realizó en la posición de decúbito lateral izquierdo, utilizando los ejes habituales: paraesternal largo y corto, apical de 2 y 4 cámaras y subxifoideo. La exploración fue llevada a cabo, bajo control electrocardiográfico continuo, antes de la implantación del dispositivo resincronizador (condiciones basales) y a los 3 meses de seguimiento, con estimulación auriculobiventricular (resincronizador conectado) y con ritmo propio del paciente (resincronizador desconectado).

Se valoraron la fracción de eyección y el diámetro telediastólico ventricular izquierdo según la fórmula de Teichholtz.

Para cuantificar la asincronía interventricular, se calculó la diferencia entre el retraso electromecánico del ventrículo derecho (tiempo en ms desde el inicio del complejo QRS al inicio del flujo pulmonar) y el izquierdo (tiempo en ms desde el inicio del complejo QRS al comienzo del flujo aórtico).

Para valorar la asincronía intraventricular se analizaron los siguientes parámetros:

- Retraso septo-pared posterior (RSP). El retraso (diferencia en ms) entre la excursión máxima del septo $\mathrm{y}$ la pared posterior, medidas en modo $\mathrm{M}$ y en el eje corto parasternal.

- Retraso septo-pared lateral (RSL). La diferencia (ms) valorada por eco-Doppler tisular entre el inicio del complejo QRS y la velocidad sistólica máxima de cada región ventricular en el eje apical de 4 cámaras.

Además, se valoraron los siguientes parámetros: la integral del flujo aórtico (mediante eco-Doppler pulsado y en el tracto de salida del ventrículo izquierdo), el patrón de llenado mitral por eco-Doppler pulsado (on- das E y A) y eco-Doppler tisular del anillo mitral (ondas E' y A'), y el cociente E/E'.

Todos los estudios ecocardiográficos fueron realizados por el mismo explorador, ajeno a la finalidad del estudio y ciego a la programación del dispositivo. Las medidas se realizaron en 3 ciclos y el valor final fue la media de dichas determinaciones.

\section{Implantación del dispositivo}

Los implantes se realizaron en el laboratorio de electrofisiología, equipado con un sistema de angiografía digital Hicor (Siemens). El procedimiento se realizó con anestesia local, mediante los accesos venosos habituales (vena subclavia y/o cefálica) y empleando las técnicas previamente descritas ${ }^{14}$.

A todos los pacientes se les realizó una angiografía del sistema venoso coronario, y se implantó el electrodo ventricular izquierdo preferentemente en su región lateral. Se accedió a la posición anterior como último recurso, en caso de imposibilidad de acceder o de presentar umbrales inaceptables de estimulación en la región lateral.

El intervalo auriculoventricular se optimizó mediante eco-Doppler y por métodos descritos anteriormente ${ }^{15}$ en todos los pacientes. El correcto funcionamiento del generador fue comprobado al alta mediante la obtención de umbrales y control radioscópico de los electrodos.

Asimismo, un observador ajeno al estudio analizó en cada paciente la diferencia entre el máximo complejo QRS estimulado y el basal.

\section{Posición del electrodo ventricular izquierdo}

Se definió zona anterior a la comprendida entre las 10 y la 1 en la posición radiológica oblicua anterior izquierda a $45^{\circ}$ y zona lateral a la comprendida entre las 2 y las 5 en la misma proyección (fig. 1).
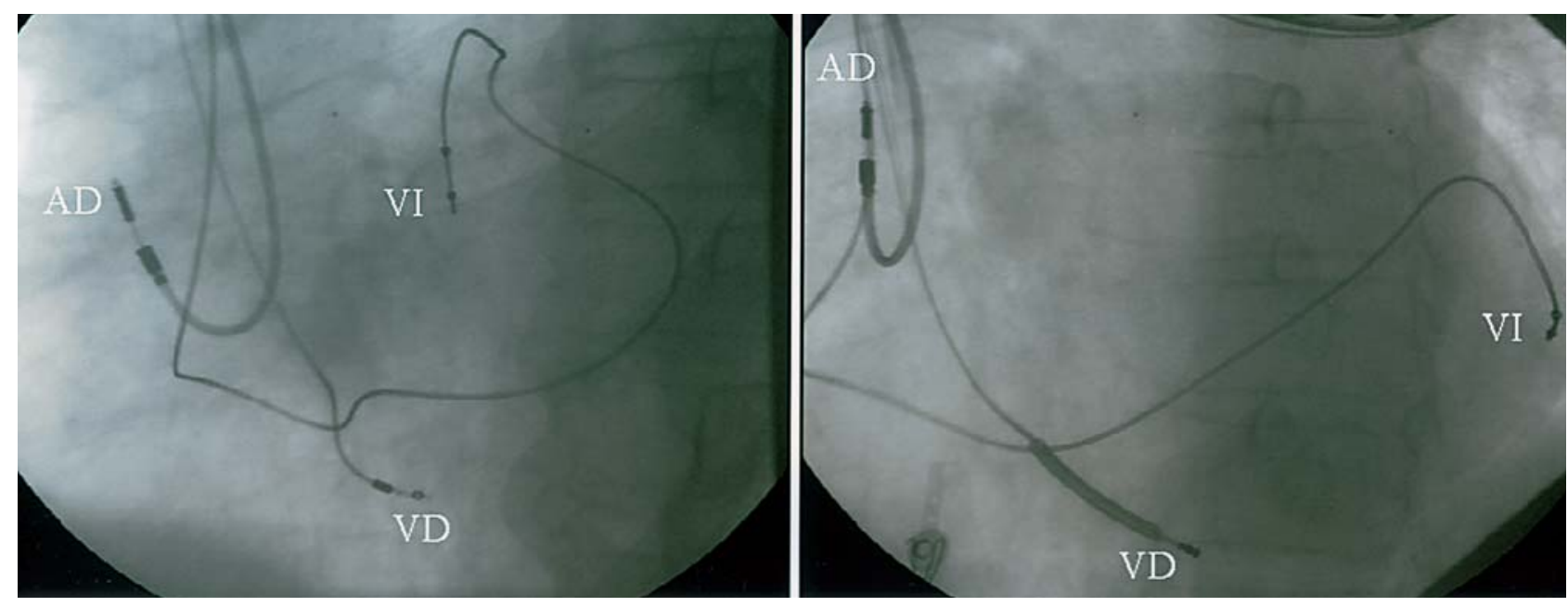

Fig. 1. Imágenes radiológicas en proyección oblicua anterior izquierda a $45^{\circ}$. El electrodo ventricular izquierdo se encuentra localizado en zona anterior (imagen izquierda) y en posición lateral (imagen derecha). AD: electrodo auricular derecho; VD: electrodo ventricular derecho; VI: electrodo ventricular izquierdo. 
TABLA 1. Características clínicas de los pacientes

\begin{tabular}{|c|c|c|c|c|}
\hline & Global & Posición lateral & Posición anterior & p (anterior frente a lateral) \\
\hline Pacientes (n) & 25 & 13 & 12 & \\
\hline Edad & $75,2 \pm 8,7$ & $72,6 \pm 9,8$ & $72,3 \pm 8,0$ & 0,95 \\
\hline Sexo (V/M) & $21 / 4$ & $11 / 2$ & $11 / 1$ & 0,98 \\
\hline \multicolumn{5}{|l|}{ Etiología (\%) } \\
\hline Isquémica & 60 & 61 & 59 & 0,89 \\
\hline No isquémica & 40 & 39 & 41 & 0,84 \\
\hline \multicolumn{5}{|l|}{ Clase funcional NYHA (\%) } \\
\hline III & 80 & 79 & 83 & 0,78 \\
\hline IV & 20 & 21 & 17 & 0,81 \\
\hline \multicolumn{5}{|l|}{ Tratamiento médico (\%) } \\
\hline IECA (ARA-II) & 100 & 100 & 100 & 1,00 \\
\hline Bloqueadores beta & 52 & 49 & 50 & 0,98 \\
\hline Diuréticos & 100 & 100 & 100 & 1,00 \\
\hline Digital & 80 & 79 & 83 & 0,84 \\
\hline Espironolactona & 60 & 61 & 59 & 0,83 \\
\hline Fracción de eyección (\%) & $23,7 \pm 6,5$ & $22,5 \pm 5,7$ & $24,3 \pm 6,8$ & 0,82 \\
\hline DTD (mm) & $72,9 \pm 8,2$ & $72,2 \pm 8,2$ & $73,8 \pm 8,1$ & 0,67 \\
\hline $\mathrm{QRS}(\mathrm{ms})$ & $159,9 \pm 29,4$ & $156 \pm 33,8$ & $161,9 \pm 25,3$ & 0,84 \\
\hline $\mathrm{PR}(\mathrm{ms})$ & $226,2 \pm 31,1$ & $237,6 \pm 28,4$ & $218,7 \pm 34,3$ & 0,28 \\
\hline
\end{tabular}

ARA-II: antagonistas de los receptores de la angiotensina II; DTD: diámetro telediastólico; IECA: inhibidores de la enzima de conversión de la angiotensina; M: mujer; V: varón.

Los datos se expresan como media \pm desviación estándar.

Se incluyó en el estudio a los primeros 13 pacientes consecutivos con el electrodo ventricular izquierdo en posición lateral o posterolateral y a los primeros 12 con el electrodo en posición anterior, también consecutivos.

Los parámetros analizados se compararon de forma global, basal y tras la resincronización en los 25 pacientes, y posteriormente dividiéndolos según la región final del ventrículo izquierdo estimulada.

\section{Análisis estadístico}

Los resultados numéricos se expresan en el estudio como media \pm desviación típica. Se estudió la normalidad de las variables cuantitativas mediante el test de Kolmogorov-Smirnov con la corrección de Lilliefors. Las diferencias en porcentajes entre grupos se compararon con la prueba exacta (paquete estadístico PEPI, de Abramson y Gahlinger, 1993-2000). Las diferencias entre las distintas variables se compararon mediante la prueba de la t de Student para muestras dependientes o independientes, según el caso. Se consideraron estadísticamente significativos los valores de $\mathrm{p}<0,05$.

\section{RESULTADOS}

En los 25 pacientes, el dispositivo estaba estimulando correctamente al alta y a los 3 meses de seguimiento. Las características demográficas y clínicas del grupo total de pacientes se presentan en la tabla 1 .
Veinte pacientes recibieron un dispositivo de resincronización Guidant TR 1241 y 5, un desfibrilador automático con capacidad de estimulación biventricular (Guidant H135 y 1823). El umbral agudo medio de estimulación fue de 1,4 $\pm 0,6$ voltios, con unas impedancias y amplitudes medias del electrodo ventricular izquierdo de $1.082 \pm 307$ ohmios y $11,1 \pm 5,7$ milivoltios, respectivamente.

A los 3 meses de seguimiento se observó una mejora en la fracción de eyección $(23,7 \pm 6,5$ frente a 27,8 $\pm 5,5 \%$ ), así como una reducción en el diámetro telediastólico del ventrículo izquierdo tras la TRC (72,9 \pm $8,2$ frente a $65,6 \pm 6,6 \mathrm{~mm})$. Ésta se asoció con un incremento en la integral del flujo aórtico $(19,6 \pm 4,3$ frente a $21,3 \pm 4,9)$ y con una disminución en el cociente E/E' del patrón de flujo mitral $(15,7 \pm 5,7$ frente a 10,7 $\pm 5,3)$. Según la localización, la fracción de eyección y la integral del flujo aórtico mejoraron significativamente sólo en el grupo de pacientes estimulados desde la región lateral, mientras que el diámetro telediastólico y el cociente E/E' mejoraron en ambas localizaciones, como se detalla en la tabla 2.

\section{Parámetros electrocardiográficos}

La duración media del complejo QRS basal fue de $159,9 \pm 29,4 \mathrm{~ms}$ y tras la estimulación biventricular, de $157,8 \pm 20,2 \mathrm{~ms}(\mathrm{p}=0,77)$. Tampoco hubo diferencias estadísticamente significativas en la anchura del complejo QRS antes y después de la implantación del dispositivo resincronizador, según la localización final 

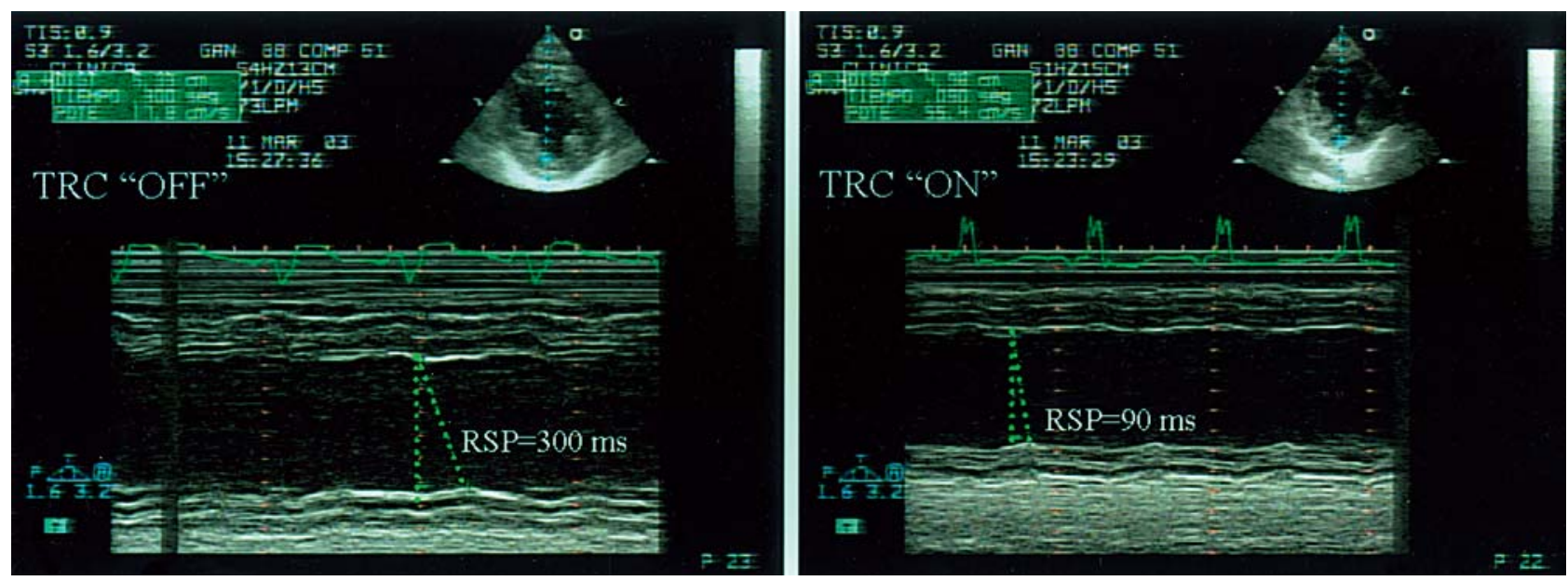

Fig. 2. Imágenes ecocardiográficas modo $\mathrm{M}$ en el eje corto paraesternal, donde se observa la disminución en el retraso entre el septo y la pared posterior con el marcapasos conectado (imagen derecha) en relación con el marcapasos desconectado (imagen izquierda). RSP: retraso septo-pared posterior; TRC «off»: sin terapia de resincronización cardíaca; TRC «on»: con terapia de resincronización cardíaca.

del electrodo ventricular izquierdo fuera lateral $(156,0$ $\pm 33,8$ frente a $154,7 \pm 18,2 \mathrm{~ms} ; \mathrm{p}=0,58)$ o anterior $(161,9 \pm 25,3$ frente a $160,9 \pm 22,6 \mathrm{~ms} ; \mathrm{p}=0,23)$.

\section{Asincronía interventricular}

La diferencia electromecánica entre el ventrículo izquierdo y el derecho fue similar cuando se comparó de forma global, en condiciones basales y a los 3 meses con el dispositivo conectado $(38,3 \pm 22,3$ frente a 31,1 $\pm 24,2 \mathrm{~ms} ; \mathrm{p}=0,35)$ y desconectado $(38,3 \pm 22,3$ frente a $37,8 \pm 21,8 \mathrm{~ms} ; \mathrm{p}=0,42$ ). Cuando se compararon los mismos parámetros teniendo en cuenta la posición del electrodo tampoco se encontraron diferencias estadísticamente significativas (tabla 3) entre las regiones anterior y lateral.

\section{Asincronía intraventricular}

Respecto a las mediciones basales, la estimulación biventricular disminuyó de forma significativa el RSP (fig. 2) en el grupo global de pacientes a los 3 meses con el dispositivo conectado $(218,3 \pm 44,7$ frente a $86,6 \pm 35,4 \mathrm{~ms} ; \mathrm{p}<0,001)$. No hubo diferencias entre el RSP basal y a los 3 meses con el dispositivo desconectado $(218,3 \pm 44,7$ frente a $211,6 \pm 34,7 \mathrm{~ms} ; \mathrm{p}=$ $0,56)$.

De la misma manera, la resincronización también se asoció a una disminución estadísticamente significativa $(\mathrm{p}=0,007)$ del RSL basal $(111,4 \pm 30,9 \mathrm{~ms})$ en relación con el RSL a los 3 meses con el dispositivo conectado (61,9 $\pm 21,3 \mathrm{~ms}$; fig. 3). El RSL basal fue similar al RSL con el dispositivo desconectado al final del seguimiento $(111,4 \pm 30,9$ frente a $115,3 \pm 28,6 ; p$ $=0,58$ ).

En cuanto a la posición del electrodo, tal y como se muestra en la tabla 3 , se observó una disminución es-
TABLA 2. Parámetros hemodinámicos medidos mediante ecocardiografía según la localización final del electrodo

\begin{tabular}{lrrr}
\hline & $\begin{array}{c}\text { Basal } \\
(\mathbf{n = 2 5})\end{array}$ & $\begin{array}{c}\mathbf{3} \text { meses } \\
(\mathbf{n}=\mathbf{2 5})\end{array}$ & $\mathbf{p}$ \\
\hline $\begin{array}{l}\text { Fracción de eyección (\%) } \\
\quad \text { Anterior }\end{array}$ & $24,3 \pm 6,8$ & $25,2 \pm 6,6$ & 0,212 \\
$\quad$ Lateral & $22,5 \pm 5,7$ & $30,5 \pm 5,2$ & 0,001 \\
$\begin{array}{l}\text { Diámetro telediastólico (mm) } \\
\quad \text { Anterior }\end{array}$ & $73,8 \pm 8,1$ & $67,3 \pm 8,4$ & 0,042 \\
$\quad$ Lateral & $72,2 \pm 8,2$ & $64,0 \pm 5,7$ & 0,035 \\
Integral aórtica & & & \\
$\quad$ Anterior & $19,5 \pm 4,1$ & $20,3 \pm 3,2$ & 0,543 \\
$\quad$ Lateral & $19,7 \pm 4,7$ & $22,2 \pm 6,1$ & 0,01 \\
Cociente E/E' & & & \\
$\quad$ Anterior & $15,6 \pm 6,3$ & $11,1 \pm 6,0$ & 0,032 \\
$\quad$ Lateral & $15,88 \pm 6,6$ & $10,5 \pm 6,3$ & 0,016 \\
\hline
\end{tabular}

Los datos se expresan como media \pm desviación estándar.

tadísticamente significativa tanto del RSP como del RSL en los pacientes con el electrodo ventricular izquierdo en posición lateral en relación con aquellos en los que el electrodo se había implantado en una localización anterior.

\section{Eventos durante el seguimiento}

De los 25 pacientes, 3 (16\%) fueron considerados no respondedores a la técnica al no presentar cambios en su clase funcional NYHA y/o una mejora > $10 \%$ en el número de metros recorridos en el test de los 6 min.

Los 3 pacientes no respondedores tenían el electrodo ventricular izquierdo implantado en una localización anterior del ventrículo izquierdo. 
Fig. 3. Cuantificación del retraso septopared lateral mediante Doppler tisular. El panel superior muestra los valores medidos antes de la resincronización, obteniéndose un retraso septo-pared lateral de $340 \mathrm{~ms}$. El panel inferior muestra los valores obtenidos tras la resincronización, con un retraso septo-pared lateral que mejora hasta $100 \mathrm{~ms}$.

DTL: Doppler tisular de pared lateral; DTS: Doppler tisular de pared septal; TRC: terapia de resincronización cardíaca.
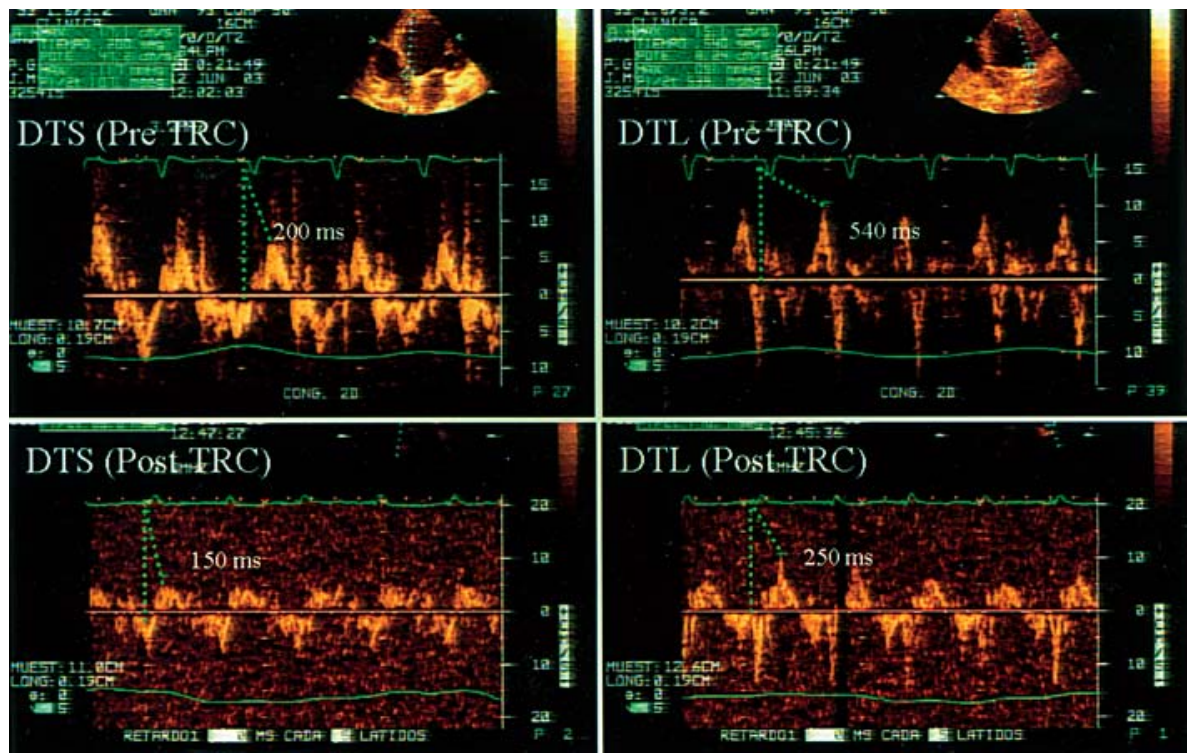

\section{DISCUSIÓN}

En el presente estudio se demuestra que existe una normalización de los parámetros RSP en modo M y RSL mediante eco-Doppler tisular tras la implantación de un dispositivo de resincronización cardíaca. Estos resultados concuerdan con los obtenidos en publicaciones de otros autores, en las que los parámetros de retraso electromecánico intraventricular (RSP y RSL) mejoran tras la resincronización e incluso parecen ser útiles a la hora de predecir un efecto clínico favorable de la TRC ${ }^{16,17}$. En una serie de 25 pacientes se evaluó, mediante eco-Doppler tisular, la velocidad sistólica máxima del septo y la pared lateral del ventrículo izquierdo, así como el retraso en la contracción entre di-

\begin{tabular}{|c|c|c|c|}
\hline & $\begin{array}{l}\text { Lateral } \\
(\mathrm{n}=13)\end{array}$ & $\begin{array}{l}\text { Anterior } \\
(\mathrm{n}=12)\end{array}$ & $\mathbf{p}$ \\
\hline \multicolumn{4}{|c|}{ Retraso septo-pared posterior (ms) } \\
\hline Basal & $189,3 \pm 51,2$ & $231,4 \pm 39,8$ & 30, \\
\hline 3 meses TRC «on» & $58,1 \pm 28,4$ & $118,0 \pm 34,1$ & 0 \\
\hline 3 meses TRC «off» & $194,1 \pm 75,0$ & $254,2 \pm 81,2$ & \\
\hline \multicolumn{4}{|c|}{ Retraso septo-pared lateral (ms) } \\
\hline Basal & $120,6 \pm 24,6$ & $112,8 \pm 21,2$ & 0 \\
\hline 3 meses TRC «on» & $39,5 \pm 13,3$ & $86,5 \pm 27,5$ & 50 \\
\hline 3 meses TRC «off» & $115,4 \pm 22,3$ & $107,0 \pm 33,3$ & \\
\hline \multicolumn{4}{|c|}{ Asincronía interventricular (ms) } \\
\hline Basal & $37,6 \pm 8,4$ & $36,5 \pm 9,3$ & 0 \\
\hline 3 meses TRC «on» & $35,3 \pm 16,1$ & $28,1 \pm 10,3$ & \\
\hline 3 meses TRC «off» & $38,1 \pm 9,8$ & $38,5 \pm 9,0$ & 0,97 \\
\hline
\end{tabular}

Los datos se expresan como media \pm desviación estándar.

TCR: terapia de resincronicación cardíaca; TRC «off»: sin terapia de resincronización cardíaca; TRC «on»: con terapia de resincronización cardíaca; 3 meses: estudio a los 3 meses de seguimiento. chas zonas. Este último parámetro mostró especificidad y sensibilidad en torno al $90 \%$ a la hora de predecir una mejora clínica con la TRC ${ }^{18}$.

Este trabajo demuestra de manera específica la influencia de la posición del electrodo ventricular izquierdo en la corrección de la asincronía intraventricular. Hasta donde nosotros conocemos, no existen datos en la bibliografía sobre la respuesta a la TRC a medio plazo según la zona ventricular estimulada. Aunque en esta serie se observa una mejoría en los parámetros medidos de asincronía intraventricular a los 3 meses con el dispositivo conectado, tanto en la posición anterior como en la lateral, cuando la región estimulada del ventrículo izquierdo es lateral en vez de anterior los efectos de la resincronización intraventricular son máximos y alcanzan una fuerte significación estadística. El hallazgo de que la mejoría de la fracción de eyección y de la integral del flujo aórtico sólo es significativa cuando la posición del electrodo es lateral parece corroborar este dato. La razón de esta mejor eficacia resincronizadora con el electrodo izquierdo en localización lateral podría encontrarse en la mayor distancia física que, en esta situación, se consigue entre ambos electrodos ventriculares, derecho e izquierdo. Este fenómeno implicaría que ambos frentes de activación se encontraran más alejados entre sí y que, por tanto, la resincronización fuera más eficiente que cuando el electrodo se implanta en la región anterior, mucho más próximo al electrodo ventricular derecho. En el mismo sentido, nuestros datos podrían ser una explicación plausible a los mejores efectos hemodinámicos agudos (en términos de dp/dt) observados con la posición lateral del electrodo ventricular izquierdo en relación con la zona anterior ${ }^{19}$. A pesar de que un reciente estudio observacional ha sugerido un efecto clínico similar en pacientes con el electrodo implantado 
en región lateral en relación con la región anterior ${ }^{20}$, no existe ningún estudio aleatorizado que haya comparado la eficacia de ambas localizaciones sobre el devenir clínico a medio plazo en pacientes sometidos a resincronización.

Queda por dilucidar la cuestión del papel que podría desempeñar la posición del electrodo en la tasa de no respondedores. A pesar de que en nuestra serie los 3 pacientes no respondedores estaban siendo estimulados desde la zona anterior, el escaso número de pacientes hace difícil extraer conclusiones al respecto. Sin embargo, a la vista de estos resultados y a pesar de ser técnicamente más complejo, parece prudente escoger una zona lateral para implantar el electrodo ventricular izquierdo siempre que sea factible ${ }^{21}$. Hay que reseñar, sin embargo, que desde el punto de vista técnico, esto puede no ser posible en más de un $30 \%$ de los casos debido a una desfavorable anatomía venosa, a la presencia de elevados umbrales de estimulación, a la inestabilidad del electrodo o a la presencia de estimulación frénica ${ }^{20}$.

En este estudio no se han obtenido diferencias en cuanto a la asincronía interventricular, la cual se ha modificado escasamente tras la implantación del dispositivo. La escasa magnitud de la asincronía interventricular observada en nuestra población podría explicar el escaso efecto de la resincronización sobre estos parámetros. Estos resultados son similares a los obtenidos en otras series ${ }^{22}$, aunque no es menos cierto que existen trabajos donde la asincronía interventricular mejora claramente durante la $\mathrm{TRC}^{23}$. Una posible teoría para explicar estas discrepancias es que la diferencia en los retrasos electromecánicos de ambos ventrículos no se correlaciona del todo bien con la asincronía de la pared lateral del ventrículo izquierdo ${ }^{24}$.

Como se ha demostrado ya en otros estudios, en nuestra serie, la TRC se asocia con una mejora en la fracción de eyección y con una disminución en el diámetro telediastólico a los 3 meses de seguimiento en el grupo estimulado desde la región lateral, resultados que traducen una probable regresión del remodelado ventricular en estos pacientes ${ }^{25}$. De manera en apariencia paradójica, a los 3 meses sigue existiendo prácticamente la misma asincronía con el dispositivo desconectado. Atendiendo a semejante cambio positivo en el remodelado ventricular, podría esperarse una cierta mejoría en la asincronía. Este fenómeno podría sugerir que, pese al remodelado positivo, la persistencia de las alteraciones de la conducción provoca que los parámetros basales de asincronía permanezcan prácticamente inalterados. Además, la TRC disminuyó el cociente E/E', cuyo valor mayor de 15 se ha asociado a un aumento de las presiones ventriculares de llenado evaluadas mediante ecocardiografía ${ }^{26}$.

En la actualidad, los criterios de asincronía son prácticamente equivalentes a presentar un bloqueo de rama izquierda con un complejo QRS > $120 \mathrm{~ms}$. Sin embargo, el bloqueo de rama izquierda no siempre conlleva una alteración en la sincronía ventricular y puede ser el resultado de otras anomalías, como defectos de conducción propiamente dichos o enlentecimiento en la propagación del impulso eléctrico en zonas isquémicas o necróticas ${ }^{27}$. De hecho, en nuestro estudio no hemos encontrado diferencias en la anchura del complejo QRS antes y después de la resincronización. Aunque una anchura de alrededor de $150 \mathrm{~ms}$ se ha relacionado con la discriminación entre respondedores y no respondedores a la TRC, tras la implantación del dispositivo esta anchura no se correlaciona con la magnitud de la resincronización ${ }^{28}$. Estos datos concuerdan con los de otros trabajos, en los que se muestra una escasa asociación entre la tasa de respondedores y el estrechamiento del complejo QRS con la estimulación biventricular ${ }^{29}$. Dada la escasa correlación entre la anchura del complejo QRS, la magnitud de la asincronía y la respuesta a la TRC, resulta tentador especular acerca del valor del eco-Doppler como interesante herramienta para el diagnóstico de asincronía, con el objetivo de disminuir el porcentaje de pacientes no respondedores. Esta potencial aplicación debería ser investigada en trabajos diseñados específicamente para este fin.

\section{Limitaciones del estudio}

Los pacientes no fueron aleatorizados, de manera que los diferentes lugares de estimulación fueron escogidos sobre la base de su accesibilidad y de los parámetros eléctricos. Este hecho limita la posibilidad de separar los efectos de la localización del electrodo de otros factores (anatómicos o de otro tipo) que impidieron el implante en posición lateral.

Los intervalos electromecánicos y eyectivos de los distintos segmentos se tomaron en ciclos cardíacos diferentes y, por tanto, están sujetos inevitablemente a la variabilidad latido a latido.

Los métodos disponibles hoy día para cuantificar la asincronía adolecen de una serie de limitaciones que es necesario reseñar. En primer lugar, el Doppler tisular no diferencia movimiento pasivo de movimiento activo en la pared miocárdica. Sería necesario realizar medidas de deformación (strain rate) para una mejor cuantificación. En segundo lugar, en la medición del RSP en modo $\mathrm{M}$, se comparan 2 pequeñas zonas de miocardio presumiendo que representan todo el movimiento de la pared. Además, puede existir un cierto grado de error a la hora de asumir que las mediciones con y sin resincronización se han realizado exactamente en la misma región. Por último, son bien conocidas las limitaciones del cálculo de la fracción de eyección con la fórmula de Teichholtz, especialmente en pacientes con alteraciones segmentarias de la contractilidad. 


\section{CONCLUSIONES}

La estimulación de la región lateral del ventrículo izquierdo se asocia con una mayor resincronización intraventricular que la de la zona anterior tras la implantación de un dispositivo de resincronización cardíaca. Los parámetros de RSP evaluados mediante ecocardiografía son de utilidad para el diagnóstico de asincronía intraventricular, ayudan a valorar los cambios de sincronía según las distintas zonas estimuladas y podrían contribuir a evaluar la eficacia de la TRC.

\section{BIBLIOGRAFÍA}

1. Leclercq C, Kass DA. Retiming the failing heart: Principles and current clinical status of cardiac resynchronization. J Am Coll Cardiol 2002;39:194-201.

2. Cazeau S, Leclercq C, Lavergne T, Walker S, Varma C, Linde C, et al. Effects of multisite biventricular pacing in patients with heart failure and intraventricular conduction delay. N Engl J Med 2001;344:873-80.

3. Abraham WT, Fisher WG, Smith AL, Delurgio DB, Leon AR, Loh E, et al. Cardiac resynchronization in chronic heart failure. $\mathrm{N}$ Engl J Med 2002;346:1845-53.

4. Auricchio A, Stellbrink C, Sacks S, Block M, Vogt J, Bakker P, et al. Long-term clinical effect of hemodynamically optimized cardiac resynchronization therapy in patients with heart failure and ventricular conduction delay. J Am Coll Cardiol 2002;39: 2026-33.

5. Gras D, Leclercq C, Tang ASL, Bucknall C, Luttikhuis HO, Kirstein-Pedersen A. Cardiac resynchroniation therapy in advanced heart failure. The multicenter InSync clinical study. Eur J Heart Fail 2002;4:311-20.

6. Ansalone G, Giannantoni P, Ricci R, Trambaiolo P, Fedele F, Santini M. Biventricular pacing in heart failure: back to basics in the pathophysiology of left bundle branch block to reduce the number of nonresponders. Am J Cardiol 2003;91(Suppl F):55-61.

7. Charles RG. Cardiac resynchronization therapy: when and for whom? Eur Heart J 2002;4(Suppl D):117-21.

8. Varma C, O'Callaghan P, Mahon NG, Hnatkova K, Mckenna W, Camm AJ, et al. Effect of multisite pacing on ventricular coordination. Heart 2002;87:322-8.

9. Ansalone G, Giannantoni P, Ricci R, Trambaiolo P, Laurenti A, Fedele F, et al. Doppler myocardial imaging in patients with heart failure receiving biventricular pacing treatment. Am Heart J 2001;142:881-96.

10. Auricchio A, Klein H, Tockman B, Sack S, Stellbrink C, Neuzner $\mathrm{J}$, et al. Transvenous biventricular pacing for heart failure: Can the obstacles be overcome? Am J Cardiol 1999;83:D136-42.

11. Butter C, Auricchio A, Stellbrink C, Fleck E, Ding J, Yu Y. Effect of resynchronization therapy stimulation site on the systolic function on heart failure patients. Circulation 2001;104:30269.

12. Blanc JJ, Etienne Y, Gilard M, Mansourati J, Munier S, Boschat $\mathrm{J}$, et al. Evaluation of different ventricular pacing sites in patients with severe heart failure: results of an acute hemodynamic study. Circulation 1997;96:3273-7.

13. Leclercq C, Cazeau S, Le Breton H, Ritter P, Mabo P, Gras D, et al. Acute hemodynamic effects of biventricular DDD pacing in patients with end-stage heart failure. J Am Coll Cardiol 1998;32: 1825-31.
14. García Bolao I, Macías A, Alegría E, Berenguel A, Gavira JJ, Azcárate $\mathrm{P}$, et al. Tratamiento de la insuficiencia cardíaca avanzada mediante estimulación biventricular. Experiencia inicial en una serie de 22 casos consecutivos. Rev Esp Cardiol 2003;56:245-52.

15. Ritter P, Padeletti L, Gillio-Meina L, Gaggini G. Determination of the optimal atrioventricular delay in DDD pacing. Comparison between echo and peak endocardial acceleration measurements. Europace 1999;1:126-30.

16. Bax JJ, Molhoek SG, Marwick TH, Van Erven L, Voogd PJ, Somer S, et al. Usefulness of myocardial tissue Doppler echocardiography to evaluate left ventricular dyssynchrony before and after biventricular pacing in patients with idiopathic dilated cardiomyopathy. Am J Cardiol 2003;91:94-7.

17. Pitzalis MV, Iacoviello M, Romito R, Massari F, Rizzon B, Luzzi $\mathrm{G}$, et al. Cardiac resynchronization therapy tailored by echocardiographic evaluation of ventricular asynchrony. J Am Coll Cardiol 2002;40:1615-22.

18. Bax JJ, Marwick TH, Van Erven L. Evaluation of resynchronization of contractile function following biventricular pacing using tissue Doppler imaging. J Am Coll Cardiol 2002;39:A356.

19. Batter C, Auriccio A, Stellbrink C, Schlegl M, Fleck E, Horsch $\mathrm{W}$, et al. Should stimulation site be tailored in the individual heart failure patient? Am J Cardiol 2000;86:K144-51.

20. Gasparini M, Mantica M, Galimberti P, Bocciolone M, Genovese L, Mangiavacchi M, et al. Is the left ventricular wall the best lead implantation site for cardiac resynchronization therapy? Pacing Clin Electrophysiol 2003;26:162-8.

21. Garrigue S, Bordachar P, Reuter S, Jais P, Kobeissi A, Gaggini $\mathrm{G}$, et al. Comparison of permanent left ventricular and biventricular pacing in patient with heart failure: prospective haemodynamic study. Heart 2002;87:529-34.

22. Nelson GS, Curry CW, Wyman BT. Predictors of systolic augmentation from left ventricular preexcitation in patients with dilated cardiomyopathy and intraventricular conduction delay. Circulation 2000;101:2703-9.

23. Zardini M, Tritto M, Bargiggia G, Forzani T, Santini M, Perego GB, et al. Analysis of clinical outcome and considerations on the selection of candidates to left ventricular resynchronization. Eur Heart J 2000;2:J16-22.

24. Kass DA. Ventricular dyssynchrony and mechanisms of resynchronization therapy. Eur Heart J 2002;4:D23-30.

25. Sutton MG, Plappert T, Abraham WT, Smith AL, Delurgio DB, Leon AR, et al. Effect of cardiac resynchronization therapy on left ventricular size and function in chronic heart failure. Circulation 2003;107:1985-90.

26. Ommen SR, Nishimura RA, Appleton CP, Miller FA, OH JK, Redfield MM, et al. Clinical utility of Doppler echocardiography and tissue Doppler imaging in the stimation of left ventricular filling pressures. A comparative simultaneous Doppler catheterization study. Circulation 2000;102:1788-94.

27. Morris-Thurgood JA, Frennaux MP. Pacing in congestive heart failure. Curr Control Trials Cardiovasc Med 2000;1:107-14.

28. Reuter S, Garrigue S, Barold S, Jais P, Hocini M, Haissaguerre $\mathrm{M}$, et al. Comparison of characteristics in responders versus nonresponders with biventricular pacing for drug resistant congestive heart failure. Am J Cardiol 2002;89:346-50.

29. Leclercq C, Faris O, Tunin R, Johnson J, Kato R, Evans F, et al. Systolic improvement and mechanical resynchronization does not require electrical synchrony in the dilates failing heart with left bundle brunch block. Circulation 2002;106:1760-3 . 